\title{
Optimizing LTE Wireless Access Networks towards Power Consumption and Electromagnetic Exposure of Human Beings
}

\author{
Margot Deruyck, Emmeric Tanghe, David Plets, Luc Martens, Wout Joseph \\ Ghent University/iMinds, Department of Information Technology, Gaston Crommenlaan 8 box 201, B-9050 Ghent, Belgium, e-mail: \\ margot.deruyck@intec.ugent.be
}

\begin{abstract}
When developing future wireless access networks, it is important to focus not only on optimizing the power consumption, but also the electromagnetic field exposure for human beings. However, optimizing towards these two parameters results in conflicting requirements on the network. In this paper, a deployment tool for future green wireless access networks is proposed, allowing four different levels of optimizations: towards power consumption, towards human exposure, towards power consumption while satisfying a certain exposure limit, and towards both power consumption and human exposure. The proposed deployment tool is capacity-based, meaning that it responds to the instantaneous bit rate required by the users in the considered area by switching base stations dynamically on and off. The tool is applied on a realistic suburban case in Ghent, Belgium to compare the four different levels of optimizations. A first compromise between optimizing towards power consumption and human exposure is obtained by optimizing towards power consumption while satisfying an exposure limit (at $2.6 \mathrm{GHz}$ ). However, there is still room for improvement especially when considering the human exposure. A better trade-off is achieved by the optimization towards power consumption and human exposure at the same time. Furthermore, the influence of the most important parameters such as the weighting factors of the fitness function, the distance between base station and general public, and between the grid points for evaluating the exposure is investigated.
\end{abstract}

Keywords: Capacity based, deployment tool, electromagnetic exposure, power consumption, wireless access network

\section{Introduction}

In the last few years, the worldwide use of wireless devices has grown considerably. Laptops, tablets, smartphones, etc. have entered into our daily life. Due to this growth, wireless access networks have also been expanding significantly in order to serve all mobile users. However, wireless access networks, and more specifically the base stations of these networks, are currently very large power consumers [1]. As it is expected that the amount of wireless devices, customers, and traffic will further increase in the coming years, it is very important to develop and implement energy-efficient wireless access networks in the near future [2,3]. In addition to this, people are becoming more and more concerned about the health effects that can be caused by the electromagnetic radiation of these networks. The Eurobarometer survey about electromagnetic fields indicates that $33 \%$ of the European respondents believes that base stations have a major effect on people's health, while

Preprint submitted to Computer Networks
$37 \%$ believes that they might have some effects on their health [4]. 6\% cannot perform an opinion on this issue. The respondents of a study performed by the European FP7 project LEXNET also see these base stations as the strongest electromagnetic field exposure sources and experience them as very dangerous [5]. A recent study in Flanders, Belgium, showed that about $4 \%$ of the respondents does not have a mobile phone [6]. Approximately $6 \%$ of these non-adopters is worried about their health. No information was provided considering possible concerns by the adopters. All these surveys show a high perception of risk and thus there is definitely a need in Europe to develop networks with a low exposure in the future. Furthermore, international organizations such as ICNIRP (International Commission on NonIonizing Radiation Protection) provide safety guidelines and national authorities define laws and norms to limit the electromagnetic fields caused by wireless network. In various countries different precautionary norms have

October 23, 2015 
been issued (e.g., in Belgium, Switzerland, etc.) [7]. Exposure awareness is thus clearly increasing and so on the other side, it is also important to minimize the exposure of human beings to these networks.

In this paper, we propose an algorithm for future green wireless access networks whereby four different levels of optimizations are possible: (i) towards power consumption only, (ii) towards human exposure only, (iii) towards power consumption while satisfying a certain exposure limit, and (iv) towards both power consumption and human exposure. Especially the trade-off between the power consumption and human exposure optimization, to the best of our knowledge, has never been done before. This trade-off is challenging because the optimization towards power consumption only results in a network with a low number of high-power base stations, while the optimization towards human exposure results in a network with a high number of low-power base station as we will shown in this paper. Furthermore, the proposed deployment tool is capacity-based, meaning that it will respond to the instantaneous bit rate requested by the user in the considered area. By using sleep modes for the base stations where little or no activity place is taking place in their cells, it is possible to respond to the actual demand and reduce power consumption and human exposure whenever possible.

The outline of this paper is as follows. In the next section, the algorithms to optimize the network with the various exposure and power consumption restrictions are discussed. Section 4 shows the results obtained with the algorithms for a realistic suburban case in Ghent, Belgium with LTE (Long Term Evolution) Advanced as wireless technology. Although we consider here LTE Advanced as wireless technology, our tool can deal with other wireless technologies as well. The performance of the different network optimizations is compared and the influence of some important parameters is investigated. Section 5 summarizes our most important conclusions.

\section{Related work}

Considering power consumption and energy efficiency in wireless access networks, various aspects have already been investigated in literature. For the power consumption of a single base station in current wireless access networks, several models are available in literature with all similar results $[1,8,9,10]$. To reduce power consumption and/or improve energy efficiency in those networks, the introduction of sleep modes $[11,12,13,14,15]$ and small cells $[16,17]$ are topics that are well investigated. However, deployment tools that focus on power consumption are rather limited. [3] proposes such a tool but only for outdoor single frequency networks.

Little work has also been done on the minimization of the human exposure when developing wireless networks. The focus of the European FP7 project LEXNET [18] is to develop effective mechanisms to reduce $50 \%$ (at least) of the public exposure and define a new metric accounting for downlink and uplink exposure, without compromising the quality of service. Energy consumption is not considered as a constraint within LEXNET. Also the planning tool of [7] has this objective but is only limited to indoor environments.

To the best of our knowledge, an algorithm that optimizes both power consumption and human exposure for outdoor cellular LTE network planning has never been proposed before.

\section{Capacity-based deployment tool}

In this section, the capacity-based deployment tool is proposed. The tool is based on the algorithm proposed in [19]. The most important changes to the algorithm of [19] are:

- An algorithm is added to optimize the network towards power consumption while satisfying a certain exposure limit, while the tool of [19] only allows towards power consumption. This makes the obtained network more realistic as the exposure from base station antennas is nowadays often regulated.

- The tool is extended with an algorithm that allows to optimize the network also the exposure only or towards both power consumption and exposure at the same time. As mentioned above, only power consumption optimization was possible in [19].

- An appropriate fitness function was created for the joint optimization. Hereby, it was also important that the individual optimizations (towards power consumption only or towards exposure only) remained possible.

- The decision for allocating a user to a base station is changed. In [19], a base station was only switched on when there was no active base station to which the user could connect. This is no longer the case as discussed in Section 3.2 because this is not the appropriate decision when optimizing towards exposure. 
- Based on this tool, it is possible to provide insights on the characteristics of a network optimized towards power consumption (whether or not satisfying a certain exposure limit), towards exposure, or to both parameters which is valuable information for the development of future green wireless access networks.

As the tool is capacity-based, realistic traffic is needed as input to the tool. So first, the generation of the traffic load is discussed. Next, the algorithm for the joint optimization towards power consumption and human exposure is discussed. Subsection 3.3 defines the fitness function used in the algorithm. In the final subsection of this section, the algorithm for the optimization towards power consumption while satisfying a certain exposure limit is discussed.

\subsection{Input of the tool}

Fig. 1 gives an overview of the different input parameters. These input parameters are the same as used by the deployment tool in [19]. Each network is of course designed for a certain area. Therefore, the area under consideration is delivered to the tool in the form of a shape file (Fig. 1 Input 1), containing detailed 3D information about the buildings (i.e., location, shape, height, etc.) in the chosen environment. The maximum number of simultaneous active users (Fig. 1 Input 2), which depends on the population density of the considered area and evolves during the day, is also provided. The distribution proposed in [19] is here considered, which is based on data retrieved from a Belgian operator. As mentioned above, the tool is capacity based, meaning that it will respond to the instantaneous bit rate requested by the users active in the network. This will be done by dynamically switching base stations on and off (i.e., using sleep modes). Therefore, it is also important to know the location of the users in the area (Fig. 1 Input 3), which is obtained by a uniform distribution (which differs from [19] where the users were located on the equidistant samples over the considered area), and the bit rate required by the user (Fig. 1 Input 4) [19]. A part of the users is assumed to make a phone call, requiring $64 \mathrm{kbps}$, while the other part is using a data connection requiring $1 \mathrm{Mbps}$. Furthermore, it is also important to know for which time stamp (Fig. 1 Input 5) the data is valid.

All the information is combined in so called traffic files (Fig. 1 Output) and provided as input to the algorithm. Due to the different distributions described above, multiple simulations need to be executed for each time interval. A value of 40 simulations is obtained

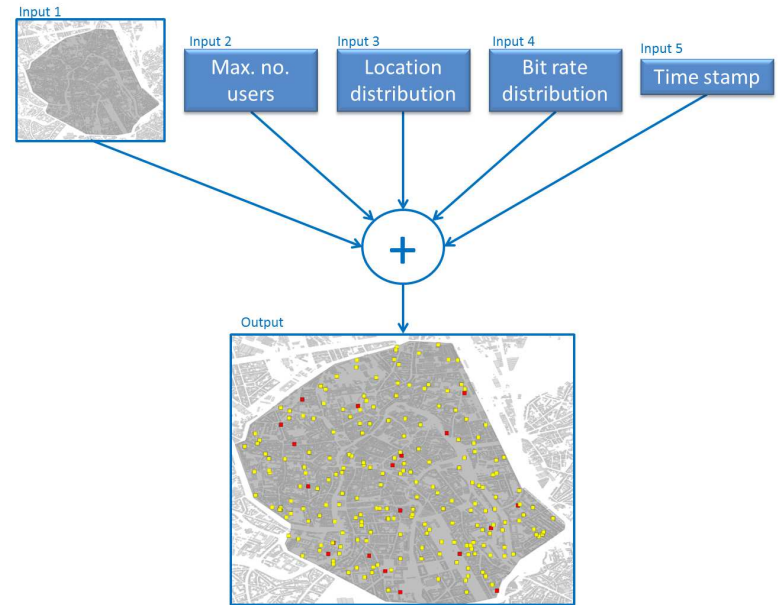

Figure 1: Schematic for generating realistic traffic (yellow squares in output $=$ users requiring $64 \mathrm{kbps}$, pink squares in output $=$ users requiring $1 \mathrm{Mbps}$ ).

to have an estimation of the $50^{\text {th }}$ and $95^{\text {th }}$ percentile for the area considered in Fig. 1 [19].

\subsection{Algorithm for the optimization towards power con- sumption and/or human exposure}

In this section, the algorithm for the optimization towards power consumption only, towards human exposure only, and towards both power consumption and human exposure is discussed. The algorithm for optimization towards power consumption while satisfying a certain exposure limit is slightly different from the others and will be discussed in a next section.

Fig. 2 gives an overview of the different steps of the algorithm. For each user active in the network (Fig. 2 Step 1), a list of possible base stations (active or by waking one up) to which the user can connect is established (Fig. 2 Step 2). A user can connect to a base station if the experienced path loss from this base station is lower than the maximum allowable path loss and if the base station can (still) offer the bit rate requested by the user. For every base station (active or asleep), we check if it can cover the user. When this is not the case, we investigate if a higher input power of the antenna can result in a user coverage. Therefore, we increase the input power of the antenna with $1 \mathrm{dBm}$ until the user is covered or until the maximum allowed input power of the antenna is reached. If the latter is the case, the base station cannot cover the user and will not be added to the list. If a base station is asleep, we start from an antenna's input power of $1 \mathrm{dBm}$. Note that if the algorithm decides in a later stage to connect the user to a different base station, the increase of the antenna's input power 
of this particular base station is nullified. To calculate the path loss, the 3D information about the buildings in the environment is considered to determine if we are in a LoS (Line-of-Sight) or NLoS (Non-Line-of-Sight) situation. The appropriate WI (Walfish Ikegami) propagation model is then used [20]. For calculating the maximum allowable path loss, the receiver SNR (Signal-toNoise-Ratio) is used. As mentioned above, each user requires a certain bit rate. To obtain this bit rate, an appropriate modulation scheme and coding rate needs to be determined. Each combination of modulation scheme and coding rate supported by LTE-Advanced corresponds with a certain receiver SNR which is the determining factor that indicates what the signal power level is for just acceptable communication quality [21]. Note that if the list is empty, it means that there is no base station available to connect this user with and we can proceed with the next user. The current user will remain uncovered. Finally note also that the order in which the users are treated can influence the results. To this end, we performed multiple simulations to minimize this effect on our results.

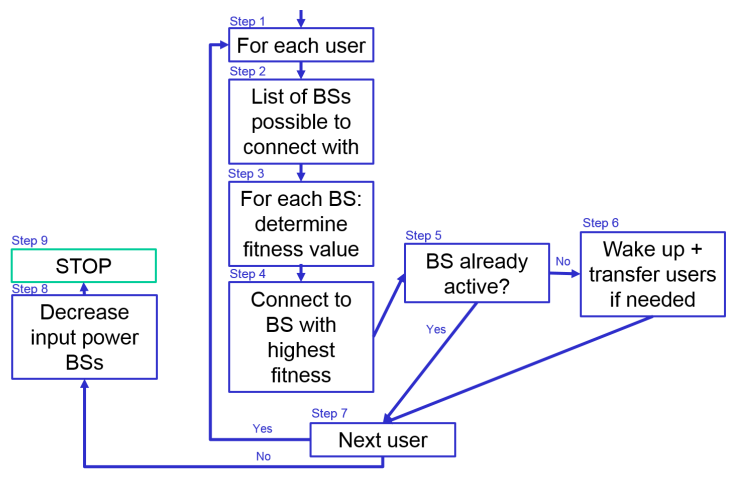

Figure 2: Flow diagram of the capacity-based deployment tool designing green wireless access networks optimized towards power consumption and global exposure

To determine which base station of the list is the best to connect with, a fitness function is used. This fitness function tells us how well a solution performs in terms of power consumption and in terms of exposure. Optimization towards power consumption only, towards human exposure only or towards both power consumption and human exposure is made possible by choosing the appropriate weighting factors (next subsection). For each base station from the list (Fig. 2, Step 3), such a fitness value will be calculated and the user will be connected to the base station obtaining the highest fitness value (Fig. 2, Step 4). Two cases are then possible: (i) the base station was already active and (ii) the base sta- tion is in sleep and needs to wake up. If (i) is the case, no further action needs to be taken and we can process to covering the next user if there are still uncovered ones (Fig. 2, Step 7). Otherwise, when (ii) is the case, we have to wake up the base station and an additional step is performed (Fig. 2, Step 6), whereby we check if we can transfer users already covered by other base stations to this 'new' base station in order to spread the load on the active base stations in the network. Transferring a user can only be done if the new base station can offer the required bit rate while obtaining a higher fitness value. Again, if possible and necessary, the input power of the base station's antenna can be increased. If all users of a certain base station can be transferred to other base stations, the base station is of course switched off. Thereafter, we can continue also with Step 7 in Fig. 2, as done in case (i). Steps 1 to 6 will be repeated until we have checked all users (Fig. 2, Step 7). The obtained solution is then further optimized by decreasing the input power of each base station when possible (Fig. 2, Step 8). Of course this is only possible, if each user connected to the base station, is still connected after decreasing the power (i.e., its experienced path loss is still lower than its maximum allowable path loss). After this step, the targeted solution is obtained and the algorithm is stopped (Fig. 2, Step 9). As mentioned before, the above described procedure needs to be repeated multiple times.

\subsection{Fitness function}

The decision to which base station a user should connect to is thus made based on the fitness value as discussed above (Fig. 2 Step 4). The fitness function is defined as follows:

$$
f_{\text {tot }}=\left(w_{1} \cdot\left(1-\frac{P}{P_{\max }}\right)+w_{2} \cdot\left(1-\frac{E_{M}}{E_{\max }}\right)\right) \cdot 100 \text { (1) }
$$

with $w_{1}$ and $w_{2}$ weight factors between 0.0 and 1.0 (boundaries included), $P$ the power consumption of the current (designed) network in Watt, $P_{\text {max }}$ the maximum power consumed by the network when all base stations are active with maximal input power of the antenna and consuming maximum power according to their settings (e.g., MIMO (Multiple-Input Multiple-Output)) in Watt, $E_{M}$ the global exposure of the current (designed) network in $\mathrm{V} / \mathrm{m}$, and $E_{\max }$ the global exposure of the network assuming that all base stations are transmitting at the highest possible power in $\mathrm{V} / \mathrm{m}$. $f_{\text {tot }}$ provides a value between 0.0 and 200.0 (boundaries included). The higher $f_{\text {tot }}$, the better the network performs in terms of power consumption and/or global exposure (i.e., the 
lower the power consumption and/or exposure is). Different optimizations for the network are obtained by choosing the weight factors as follows:

- Power consumption: $w_{1}=1$ and $w_{2}=0$

- Exposure: $w_{1}=0$ and $w_{2}=1$

- Both power consumption and exposure: $w_{1}=0.5$ and $w_{2}=0.5$

$P$ and $P_{\max }$ are determined by using the power consumption model for different base station types from [1]. Only macrocell base stations are considered in this study. Note that we have here considered the electric-field values by using $\frac{E_{M}}{E_{m a}}$ in Eq. (1) as legislation in Belgium is explicitly related to electric-field values and more understandable for authorities. It is also possible to to use $\left(\frac{E_{M}}{E_{\max }}\right)^{2}$ in Eq. (1) which is related to power values.

To determine $E_{M}$ and $E_{\text {max }}$, the global exposure $E_{M}$ (in $\mathrm{V} / \mathrm{m}$ ) is considered. $E_{M}$ is defined as a weighted average of the median electric-field strength $E_{50}$ and the $95 \%$-percentile value of the field strengths $E_{95}$ [7]:

$$
E_{M}=\frac{w_{3} \cdot E_{50}+w_{4} \cdot E_{95}}{w_{3}+w_{4}}
$$

with $w_{3}$ and $w_{4}$ weighting factors for the $50 \%$-percentile value $E_{50}$ in $\mathrm{V} / \mathrm{m}$ and the $95 \%$-percentile value $E_{95}$ in $\mathrm{V} / \mathrm{m}$ respectively. It is important to optimize not only towards the median value $\left(E_{50}\right)$ but also to limit the higher exposure values $\left(E_{95}\right)$. A low value of $E_{50}$ in combination with a high spread of values still results in a high overall exposure. Therefore, we have included both parameters $E_{50}$ and $E_{95}$ with equal importance in Eq. (2). A value of 0.5 is used for both $w_{3}$ and $w_{4}$. To define $E_{50}$ and $E_{95}$, a grid is used with a fixed distance between two different grid points in both $\mathrm{x}$ - and $\mathrm{y}$-direction as shown in Fig. 3. For each grid point, the path loss experienced from each active base station in the network is determined. Based on this path loss, it is possible to define the electric field $\mathrm{E}$ (in $\mathrm{V} / \mathrm{m}$ ) caused by the base station in that grid point as follows [7]:

$$
E=10^{\frac{E I R P-43.15+20 \cdot \log _{10}(f)-P L}{20}}
$$

with EIRP the Equivalent Isotropically Radiated Power in $\mathrm{dBm}, f$ the frequency in $\mathrm{MHz}$, and $P L$ the experienced path loss in $\mathrm{dB}$. To determine the path loss $P L$ experienced in a grid point, the same approach as described above to determine the path loss experienced by a user from a certain base station is used. The electric field caused by all contributing sources at a certain location can then be determined as follows (in $\mathrm{V} / \mathrm{m}$ ) [7]:

$$
E_{t o t}=\sqrt{\sum_{i=1}^{n} E_{i}^{2}}
$$

with $n$ the number of active base stations in the network, and $E_{i}$ the electric field caused by base station $i$ and determined by Eq. (3). For every grid point, an $E_{t o t}$ value will be determined. If we have thus $m$ grid points, we have $m E_{t o t}$ values. The $E_{50}$ (resp. $E_{95}$ ) value is then determined by taking the 50\%-percentile (resp. 95\%percentile) over these $m E_{t o t}$ values. Once we know $E_{50}$ and $E_{95}, E_{M}$ is calculated by Eq. (2). Note that only the base stations of the network that need to be optimized are taken into account. In order to obtain the real exposure a user experiences in the considered area, all base stations (of the different operators) active in the area should be taken into account.

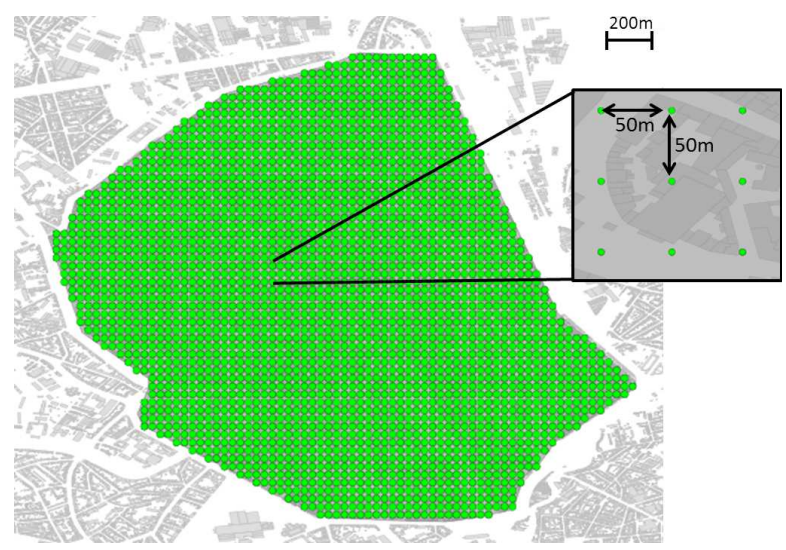

Figure 3: Grid used to evaluate the exposure in the area of interest.

\subsection{Algorithm for optimization towards power con- sumption while satisfying a certain exposure limit}

In this section, the algorithm for optimizing the network towards power consumption while satisfying a certain exposure limit per antenna is proposed. This algorithm differs slightly from the one optimizing both power consumption and exposure as we will discuss. The data of Section 3.1 is again provided as input to the algorithm, along with a predefined exposure limit $E_{\text {lim }}$ which is the maximally allowed electric field strength (in $\mathrm{V} / \mathrm{m}$ ) per antenna. The first step of the algorithm is, as shown in Fig. 4, to determine the maximum EIRP, here denoted as $E I R P_{\max }$ (in $\mathrm{dBm}$ ), based on the predefined exposure limit as follows [7]:

$$
E I R P_{\text {max }}=43.15+20 \cdot \log _{10} \frac{E_{\text {lim }}}{f}+P L_{d}
$$


with $E_{\text {lim }}$ the maximally allowed electric field strength (in $\mathrm{V} / \mathrm{m}$ ), $f$ the frequency in $\mathrm{MHz}$, and $P L_{d}$ the path loss at a distance $d$ experienced from that base station (in $\mathrm{dB}$ ). The distance $d$ defines the minimal separation between the human from the base stations that will be installed.

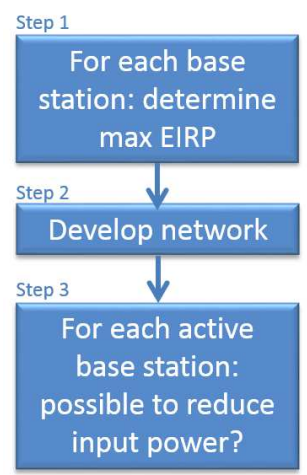

Figure 4: Flow diagram of the capacity-based deployment tool for designing green wireless access networks optimized towards power consumption while satisfying a certain exposure limit per antenna.

Once the maximum EIRP for each antenna is known, the network can be developed (Fig. 4, Step 2). Here, no fitness function is used, but instead, the user is connected to the base station that can offer the bit rate requested by the user for the lowest path loss (which is of course on its turn lower than the maximum allowable path loss). The algorithm tries to connect the users, if possible, to an active base station as waking a new base station up results in an extra power consumption between $1352 \mathrm{~W}(2105 \mathrm{~W}$ at $1 \mathrm{dBm}-753 \mathrm{~W}$ for sleep mode) and $3228 \mathrm{~W}(3980 \mathrm{~W}$ active state at $43 \mathrm{dBm}$ $753 \mathrm{~W}$ for sleep mode) for 4x4 MIMO, while connecting a user to an active base station results in an extra power consumption between $0 \mathrm{~W}$ (when the user can be covered without increasing the input power of the already active base station) and $1876 \mathrm{~W}$ (when the input power of the active base station has to be increased from $1 \mathrm{dBm}$ to its maximum power of $43 \mathrm{dBm}$ ) $[1,21]$.

In the final step of the algorithm (Fig. 4, Step 3), the input power of the active base stations can be decreased if possible. This can of course only be done when the users connected to the base stations can still be served after decreasing the power.

\section{Results}

In this section, the results are shown obtained with the algorithms described above. First, the selected scenario and assumptions are described. Next, we study how the different parameters of the algorithm (weighting factors of Eq. (1), the minimal distance $d$ between base station and general public, and the distance $d_{g}$ between the grid points) influence the results. In a last part, the comparison between the different optimizations (power consumption only, power consumption while satisfying a certain exposure limit, exposure only, and joint optimization of power consumption and exposure) is investigated.

\subsection{Selected scenario and assumptions}

The considered area is shown in Fig. 5. This is an outdoor suburban area in Ghent, Belgium of approximately $6.85 \mathrm{~km}^{2}$. The locations (75 in total) of the base stations are indicated by red squares and are existing base stations from Belgian mobile operators. We study the worst-case scenario, so the time stamp of the day with the maximum number of simultaneously active users (i.e., 5-6 p.m. time interval with 226 users) is considered [19]. 205 users require a bit rate of $1 \mathrm{Mbps}$ (i.e., data traffic), while the other 21 users require only $64 \mathrm{kbps}$ (i.e., voice traffic). These assumptions are based on confidential data obtained from a Belgian mobile operator. We assume LTE-Advanced as wireless technology with a frequency of $2.6 \mathrm{GHz}$ and a bandwidth of $5 \mathrm{MHz}$, supporting 4x4 MIMO. For all the other link budget parameters, we refer to Table 1 . These parameters are retrieved from specifications and/or typical values proposed by the operators themselves $[1,21]$. More information about the individual parameters can be found in [21].

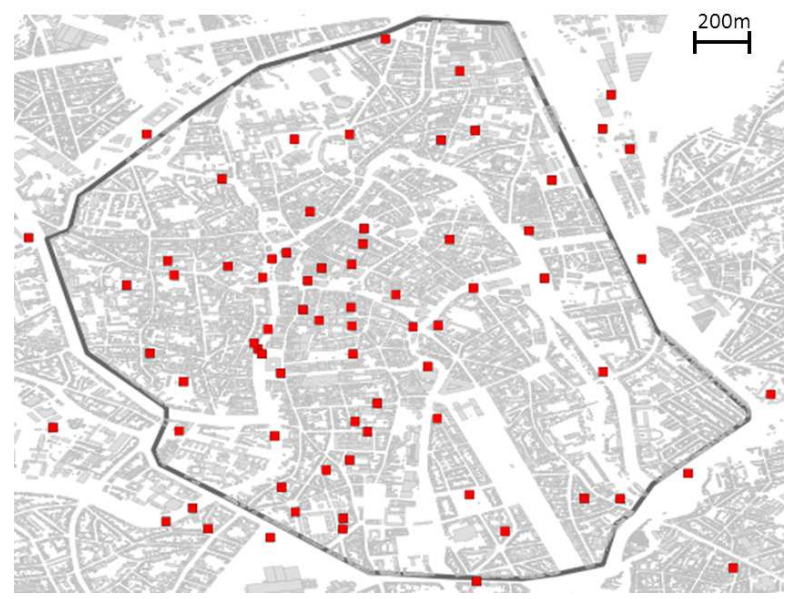

Figure 5: The selected suburban area of $6.85 \mathrm{~km}^{2}$ and the base station locations in Ghent, Belgium. 


\begin{tabular}{|l|c|}
\hline Parameter & Value \\
\hline Frequency & $2.6 \mathrm{GHz}$ \\
\hline Maximum input power antenna & $43 \mathrm{dBm}$ \\
\hline Antenna gain base station & $18 \mathrm{~dB}$ \\
\hline Antenna gain mobile station & $0 \mathrm{~dB}$ \\
\hline Soft handover gain & $0 \mathrm{~dB}$ \\
\hline Feeder loss base station & $2 \mathrm{~dB}$ \\
\hline Feeder loss mobile station & $0 \mathrm{~dB}$ \\
\hline Fade margin & $10 \mathrm{~dB}$ \\
\hline Yearly availability & $99.995 \%$ \\
\hline Cell interference margin & $2 \mathrm{~dB}$ \\
\hline User interference margin & $0 \mathrm{~dB}$ \\
\hline Bandwidth & $5 \mathrm{MHz}$ \\
\hline Receiver SNR & $1 / 3 \mathrm{QPSK}=-1.5 \mathrm{~dB}$, \\
& $1 / 2 \mathrm{QPSK}=3 \mathrm{~dB}$, \\
& $2 / 3 \mathrm{QPSK}=10.5 \mathrm{~dB}$, \\
& $1 / 216-\mathrm{QAM}=14 \mathrm{~dB}$, \\
& $2 / 316-\mathrm{QAM}=19 \mathrm{~dB}$, \\
& $4 / 516-\mathrm{QAM}=23 \mathrm{~dB}$, \\
& $2 / 364-\mathrm{QAM}=29.4 \mathrm{~dB}$ \\
\hline Used subcarriers & 301 \\
\hline Total subcarriers & 512 \\
\hline Noise figure mobile station & $8 \mathrm{~dB}$ \\
\hline Implementation loss mobile station & $0 \mathrm{~dB}$ \\
\hline Height mobile station & $1.5 \mathrm{~m}$ \\
\hline Coverage requirement & $90 \%$ \\
\hline Shadowing margin & $13.2 \mathrm{~dB}$ \\
\hline
\end{tabular}

Table 1: Link budget table of the LTE-Advanced macrocell base station $[1,21]$.

For evaluating the exposure, we use a grid with a distance of $50 \mathrm{~m}$ (unless mentioned otherwise) between two different points in both $\mathrm{x}$ - and $\mathrm{y}$-direction as shown in Fig. 3. This results in a total of 2737 grid points. The exposure of a certain base station will not be taken into account for the grid points within a distance $d$ of $25 \mathrm{~m}$ (unless mentioned otherwise) from that specific base station as it is assumed that no human will be closer to the base station than the predefined distance.

For evaluating the power consumption, the model of $[1,21]$ is used for the base station in operational state (resulting in a maximum power consumption of $3980.6 \mathrm{~W}$ assuming 4x4 MIMO), while it is assumed that the base station consumes $45 \%$ of their maximal SISO (Single-Input Single-Output) power consumption (i.e., $45 \%$ of $1674 \mathrm{~W}=753.3 \mathrm{~W}$ ) during sleep mode as proposed by [22]. Note however that the power consumption of a single base station can be lower in the solution obtained with our algorithm, as the algorithms tries to reduce the input power of the antenna, if possible, in order to lower the power consumption. Tuning the input power of the antenna and the use of sleep mode can clearly also reduce the exposure from a certain base station. A base station has e.g. an electric field strength of (maximum) $1.4 \mathrm{mV} / \mathrm{m}$ at a distance of $1 \mathrm{~m}$ (for a certain configuration) and an input power of $43 \mathrm{dBm}$, while the same base station has an electric field strength of (maximum) $0.01 \mathrm{mV} / \mathrm{m}$ at the same distance fir an input power of $1 \mathrm{dBm}$. The reduction increases when considering sleep modes as we assume that there is no radiation during sleep mode.

The influence on the power consumption and the global exposure will be compared for four different networks: (i) one optimized towards power consumption (algorithm of Section 3.2 with $w_{1}=1 \& w_{2}=0$ ), (ii) one optimized towards global exposure (algorithm of Section 3.2 with $w_{1}=0 \& w_{2}=1$ ), (iii) one optimized towards power consumption while satisfying a certain exposure limit (algorithm from Section 3.4), and finally, (iv) one optimized towards both power consumption and global exposure (algorithm of Section 3.2 with $w_{1}$ $\left.=0.5 \& w_{2}=0.5\right)$. When satisfying a certain exposure limit, an exposure limit of $4.48 \mathrm{~V} / \mathrm{m}$ per antenna at $2.6 \mathrm{GHz}$ is considered (mandated by the Flemish exposure norm [7]). Compared to the ICNIRP guidelines which recommend a limit of $61 \mathrm{~V} / \mathrm{m}$ for the considered frequency [23], the Flemish limit is restrictive. However, also other countries have different limits. In Switzerland, a limit of $6 \mathrm{~V} / \mathrm{m}$ at $2.6 \mathrm{GHz}$ per base station is obtained for sensitive areas i.e., living rooms, school rooms, kindergarten, hospitals, nursing homes, places of employment, childrens and school playgrounds [24]. Italy has a limit of $6 \mathrm{~V} / \mathrm{m}$ [7]. Brussels has a limit of $6 \mathrm{~V} / \mathrm{m}$ but for all sources instead of per base station, valid at all public availability places [7, 24]. In China, a value of $12 \mathrm{~V} / \mathrm{m}$ is obtained [7]. The Netherlands follow ICNIRP and thus has a limit of $61 \mathrm{~V} / \mathrm{m}$ for all sources [24]. As already mentioned, for each optimization, 40 simulations are performed.

\subsection{Varying the weighting factors $w_{1}$ and $w_{2}$}

Fig. 6 shows the influence of varying the $w_{1}$ and $w_{2}$ parameter of Eq. (1). In each figure, the influence of varying the $w_{2}$ factor for a fixed $w_{1}$ on the power consumption and the global exposure is shown. The blue solid line represents the $50 \%$-percentile along with the obtained $90 \%$ confidence interval and the red dashed line represents the $95 \%$-percentile.

Fig. 6 shows that for a fixed $w_{1}$, a higher $w_{2}$ results in a lower global exposure $E_{M}$ (in $\mathrm{mV} / \mathrm{m}$ ), but also as expected in a higher power consumption $P C$ (in $\mathrm{kW})$. For example, for $w_{1}=0.2$ and $w_{2}=0.2$, a power consumption of $100 \mathrm{~kW}\left(p_{50}\right)$ and a global exposure of $163.1 \mathrm{mV} / \mathrm{m}\left(p_{50}\right)$ is obtained versus $108 \mathrm{~kW}$ and $127.7 \mathrm{mV} / \mathrm{m}$ for $w_{1}=0.2$ and $w_{2}=0.8$. Analogously, 

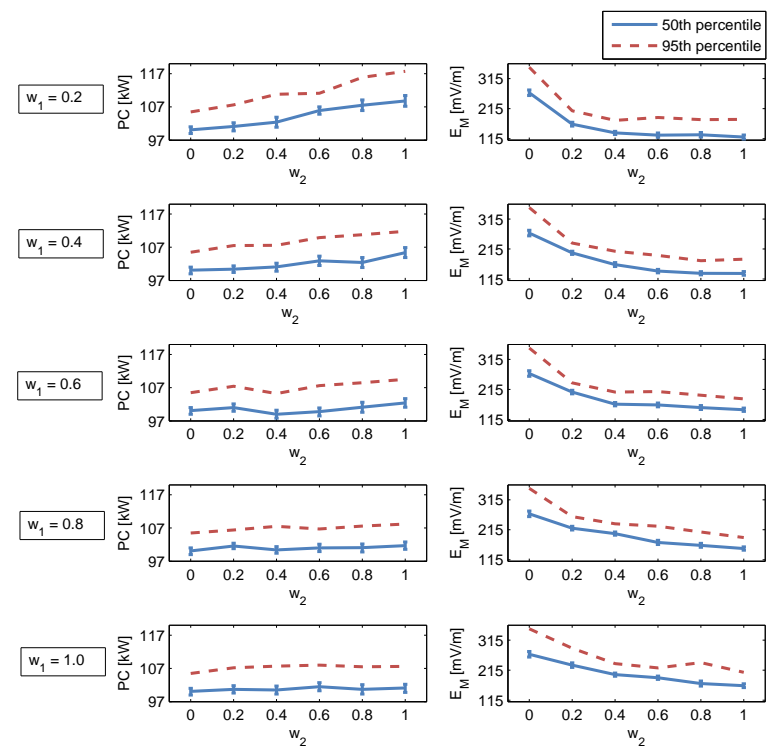

Figure 6: Influence of the $w_{1}$ and $w_{2}$ weight factors of Eq. (1) on the performance of the developed LTE-Advanced network.

a higher $w_{1}$ for a fixed $w_{2}$ results in a lower power consumption but also in a higher global exposure. For example, for $w_{1}=0.2$ and $w_{2}=0.8$, a power consumption of $108 \mathrm{~kW}\left(p_{50}\right)$ and a global exposure of $127.7 \mathrm{mV} / \mathrm{m}$ $\left(p_{50}\right)$ is obtained versus $100 \mathrm{~kW}$ and $163.1 \mathrm{mV} / \mathrm{m}$ for $w_{1}=0.8$ and $w_{2}=0.8$. Note however that the influence of varying weight factors is limited when optimizing towards both power consumption and global exposure. A minimum value of $100 \mathrm{~kW}\left(p_{50}, w_{1}=0.6\right.$ and $\left.w_{2}=0.4\right)$ and $126.7 \mathrm{mV} / \mathrm{m}\left(p_{50}, w_{1}=0.2\right.$ and $\left.w_{2}=0.6\right)$ is obtained versus a maximum value of $108 \mathrm{~kW}\left(p_{50}, w_{1}=\right.$ 0.2 and $\left.w_{2}=0.8\right)$ and $231.9 \mathrm{mV} / \mathrm{m}\left(p_{50}, w_{1}=0.8\right.$ and $\left.w_{2}=0.2\right)$. The higher one of the two weight factors, the less influence of course the other weight factor has.

In conclusion, the ratio between the two weight factors $w_{1}$ and $w_{2}$ is decisive for the obtained optimization. The higher this ratio, the more the network will be optimized towards global exposure. Similarly, the lower this ratio, the more the network will be optimized towards power consumption. When the ratio $\frac{w_{1}}{w_{2}}$ increases (respectively decreases) with $25 \%$, the power consumption increases (respectively decreases) with $1.2 \%$ and the global exposure decreases (respectively increases) with $6.7 \%$.

\subsection{Influence of the minimal distance $d$ between base station and the general public}

When developing the network (and evaluating the global exposure), a minimal distance $d$ between the base station and the human is assumed. Within distance $d$ of the base station, it is assumed that no general public will be present and that the predefined norm does not have to be satisfied. Here, we investigate how this assumption influences our results. For this study, the network optimized towards power consumption satisfying $4.48 \mathrm{~V} / \mathrm{m}$ at $2.6 \mathrm{GHz}$ per antenna is considered. Three different values for $d$ are investigated: $5 \mathrm{~m}, 15 \mathrm{~m}$, and $25 \mathrm{~m}$. All other parameters remain the same as described above. Table 2 lists the obtained results.

The larger the proximity $d$ of the general public to the base station, the less base stations are active: 34 for $5 \mathrm{~m}, 23$ for $15 \mathrm{~m}$, and 20 for $25 \mathrm{~m}$. This is due to the fact that when a human is closer to the base station, he/she experiences a higher electric field and thus the algorithm will limit the base station's EIRP (and thus the antenna's input power) more. This limitation in EIRP results in a lower range per base station and thus more base stations will be needed, resulting in a similar power consumption for the different scenarios. However, the global exposure is lower for a lower $d$ as for a lower $d$ the antenna's input power is more limited resulting in a network with a higher number of low-power base stations. A maximum value of $85 \mathrm{mV} / \mathrm{m}$ is found for $5 \mathrm{~m}$, while for $25 \mathrm{~m}$ a maximum value of $293 \mathrm{mV} / \mathrm{m}$ is obtained. Note again, that the user coverage for $d=5 \mathrm{~m}$ is significantly lower than for $15 \mathrm{~m}$ and $25 \mathrm{~m}$. Due to the limitation of the antenna's input power, it is not possible anymore to cover all users (last column of Table 2).

A value of $25 \mathrm{~m}$ will be used from now on.

\subsection{Influence of the distance $d_{g}$ between the grid points}

Another input parameter for the above described algorithms is the grid size to evaluate the global exposure of the developed network. In this section, we investigate the influence of the distance $d_{g}$ between two grid points. Three distances are considered: $10 \mathrm{~m}, 30 \mathrm{~m}$, and $50 \mathrm{~m}$. The network optimized towards power consumption satisfying $4.48 \mathrm{~V} / \mathrm{m}$ is again considered. All other parameters remain the same as mentioned above. Table 3 lists the results.

Table 3 shows that the distance between the grid points does not influence the performance parameters (power consumption and global exposure) significantly. This is quite obvious as the purpose of the grid is only to evaluate the global exposure. It does not have a direct influence on the development of the network as for example, the input power of the antenna. 


\begin{tabular}{|l|c|c|c|c||c|c|c||c|}
\hline Scenario & \#BS & $\begin{array}{c}\mathrm{PC} \\
p_{50} \\
{[-]}\end{array}$ & $\begin{array}{c}\mathrm{PC} \\
p_{95} \\
{[\mathrm{~kW}]}\end{array}$ & $\begin{array}{c}C I_{P C} \\
{[\mathrm{~kW}]}\end{array}$ & $\begin{array}{c}E_{M} \\
p_{50} \\
{[\mathrm{~kW}]}\end{array}$ & $\begin{array}{c}E_{M} \\
p_{95} \\
{[\mathrm{mV} / \mathrm{m}]}\end{array}$ & $\begin{array}{c}C I_{E_{M}} \\
{[\mathrm{mV} / \mathrm{m}]}\end{array}$ & $\begin{array}{c}\text { Cov. } \\
\text { users } \\
{[\%]}\end{array}$ \\
\hline$d=25 \mathrm{~m}$ & 21 & 104.7 & 109.5 & \pm 1.0 & 259.0 & 293.4 & \pm 5.2 & 100 \\
\hline$d=15 \mathrm{~m}$ & 23 & 96.3 & 98.9 & \pm 0.8 & 176.9 & 193.8 & \pm 2.4 & 99.6 \\
\hline$d=5 \mathrm{~m}$ & 34 & 103.9 & 108.7 & \pm 0.9 & 80.2 & 84.7 & \pm 1.1 & 85.3 \\
\hline
\end{tabular}

Table 2: Influence of the minimal distance $d$ between base station and human on the performance of the network.

\begin{tabular}{|c|c|c|c|c|c|c|c|c|}
\hline Scenario & $\begin{array}{r}\text { \#BS } \\
{[-]} \\
\end{array}$ & $\begin{array}{c}\mathrm{PC} \\
p_{50} \\
{[\mathrm{~kW}]}\end{array}$ & $\begin{array}{c}\mathrm{PC} \\
p_{95} \\
{[\mathrm{~kW}]}\end{array}$ & $\begin{array}{l}C I_{P C} \\
{[\mathrm{~kW}]}\end{array}$ & $\begin{array}{c}E_{M} \\
p_{50} \\
{[\mathrm{mV} / \mathrm{m}]}\end{array}$ & $\begin{array}{c}E_{M} \\
p_{95} \\
{[\mathrm{mV} / \mathrm{m}]}\end{array}$ & $\begin{array}{c}C I_{E_{M}} \\
{[\mathrm{mV} / \mathrm{m}]}\end{array}$ & $\begin{array}{c}\text { Cov. } \\
\text { users } \\
{[\%]}\end{array}$ \\
\hline$d_{g}=$ & 21 & 104.7 & 109.5 & \pm 1.0 & 259.0 & 293.4 & \pm 5.2 & 100 \\
\hline$d_{g}=30 \mathrm{~m}$ & 21 & 104.7 & 109.5 & \pm 1.0 & 259.1 & 286.6 & \pm 5.4 & 100 \\
\hline$d_{g}=10 \mathrm{~m}$ & 21 & 104.7 & 109.5 & \pm 1.0 & 259.2 & 288.7 & \pm 4.7 & 100 \\
\hline
\end{tabular}

Table 3: Influence of the distance $d_{g}$ between the grid points on the performance of the network.

A value of $50 \mathrm{~m}$ will be used from now on.

\subsection{Comparison of the different optimizations}

Table 4 lists the different performance parameters (number of active base stations \#BS, power consumption $P C$, global exposure $E_{M}$, and percentage of served users) for the four different optimizations. For each parameter (except for the number of active base stations and the user coverage for which we only show the $50 \%$ percentile), the $50 \%$ - $\left(p_{50}\right)$, the $95 \%$-percentile $\left(p_{95}\right)$, and the $90 \%$ confidence interval $C I$ calculated over the 40 simulations is shown. Note that the power consumption $P C$ equals the power consumption of the active base station in the network plus the power consumed by the base stations in sleep mode.

When optimizing towards power consumption only, a power consumption of $100.1 \mathrm{~kW}$ is obtained for the $50 \%$-percentile and $105.5 \mathrm{~kW}$ for the $95 \%$-percentile. [19] proposes an algorithm focusing solely on power consumption. For $4 \times 4 \mathrm{MIMO}$ and the same scenario as considered here, a value of $60.4 \mathrm{~kW}$ for the $50 \%$ percentile and $65.4 \mathrm{~kW}$ for the $95 \%$-percentile is obtained. However, [19] assumes that the base station consumes no power at all during sleep mode. Considering a power consumption of $753 \mathrm{~W}$ during sleep as discussed above, a value of $101.0 \mathrm{~kW}$ for the $50 \%$-percentile and $105.0 \mathrm{~kW}$ for the $95 \%$-percentile is obtained. The results obtained with the two algorithms differ not significantly from each other.

Comparing the optimization towards power consumption and towards global exposure, we found that optimizing towards power consumption results in a network with a low number of base stations (i.e., 20) while a network optimized towards global exposure leads to a high number of base stations (i.e., 38). As the user coverage of both networks is $100 \%$, one would expect that the base stations in the network optimized towards power consumption are high-powered, while the network optimized towards global exposure consists of low-power base stations. This is confirmed by Fig. 7 . Here, an overview of how many times each possible input power of the base station's antenna occurs in the network optimized towards power consumption (in blue) and in the network optimized towards global exposure (in yellow) is provided. For the network optimized towards power consumption, only the higher input powers are used (between $25 \mathrm{dBm}$ and $43 \mathrm{dBm}$ ), while the network optimized towards global exposure uses almost all possible input powers from $1 \mathrm{dBm}$ to $43 \mathrm{dBm}$. We conclude that the network optimized towards power consumption results in a network with a low number of high-power base stations, while the network optimized towards global exposure consists of a high number of low-power base stations. Due to the low number of base stations in the network optimized towards power consumption, a low overall power consumption is obtained (i.e., $100 \mathrm{~kW}$ versus $120.9 \mathrm{~kW}$ for $p_{50}$ in Table 4), while the fact that the base stations are high-powered is responsible for a global exposure that is 2.5 times higher compared to the network optimized towards global exposure (i.e., $268.2 \mathrm{mV} / \mathrm{m}$ versus $104.2 \mathrm{mV} / \mathrm{m}$ for $p_{50}$ in Table 4). However, note that this conclusion is valid for a network consisting of macrocell base stations only. When introducing femtocell base stations in the network, the obtained results can differ as it is easier to bring the femtocell base station close to the end user 


\begin{tabular}{|l|c|c|c|c||c|c|c||c|}
\hline Scenario & \#BS & $\begin{array}{c}\mathrm{PC} \\
p_{50} \\
{[-]}\end{array}$ & $\begin{array}{c}\mathrm{PC} \\
p_{95} \\
{[\mathrm{~kW}]}\end{array}$ & $\begin{array}{c}C I_{P C} \\
{[\mathrm{~kW}]}\end{array}$ & $\begin{array}{c}E_{M} \\
p_{50} \\
{[\mathrm{mV} / \mathrm{m}]}\end{array}$ & $\begin{array}{c}E_{M} \\
p_{95} \\
{[\mathrm{mV} / \mathrm{m}]}\end{array}$ & $\begin{array}{c}C I_{E_{M}} \\
{[\mathrm{mV} / \mathrm{m}]}\end{array}$ & $\begin{array}{c}\text { Cov. } \\
\text { users } \\
{[\%]}\end{array}$ \\
\hline Power consumption & 20 & 100.1 & 105.5 & \pm 0.9 & 268.2 & 353.2 & \pm 9.3 & 100 \\
\hline $\begin{array}{l}\text { Power consumption } \\
\text { satisfying 4.48 V/m }\end{array}$ & 21 & 104.7 & 109.5 & \pm 1.0 & 259.0 & 293.4 & \pm 5.2 & 100 \\
\hline $\begin{array}{l}\text { Power consumption \& } \\
\text { exposure }\end{array}$ & 23 & 101.1 & 107.6 & \pm 1.1 & 163.1 & 207.7 & \pm 6.4 & 100 \\
\hline Exposure & 38 & 120.9 & 127.1 & \pm 1.7 & 104.2 & 175.0 & \pm 9.1 & 100 \\
\hline
\end{tabular}

Table 4: Overview of the performance of the developed networks (40 simulations) when optimizing towards power consumption, towards power consumption while satisfying a certain exposure limit, towards global exposure, and towards both power consumption and global exposure (\#BS $=$ number of base stations, $P C=$ power consumption, $E_{M}=$ global exposure, $C I_{P C}=$ power consumption confidence interval, and $C I_{E_{M}}=$ global exposure confidence interval).

and it is also possible to install far more femtocell base stations for the power consumption of 1 macrocell base station [19].

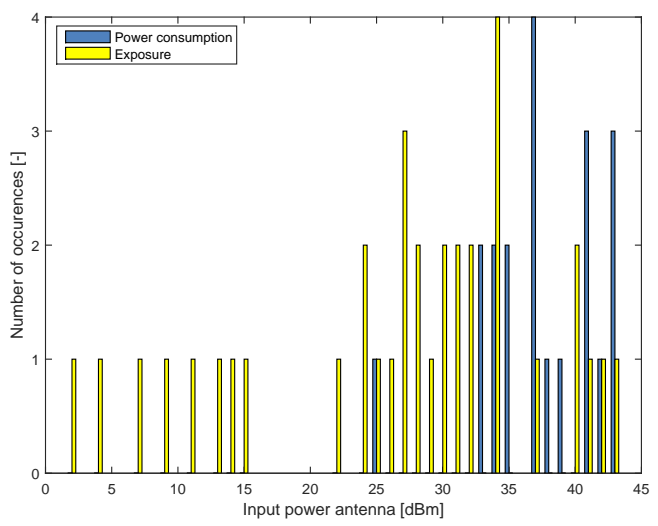

Figure 7: Overview of the number of occurrences of the possible input powers for the base station's antenna when optimizing towards power consumption (in blue) and towards global exposure (in yellow).

A first trade-off between optimizing towards power consumption and global exposure is obtained when optimizing the network towards power consumption while satisfying a certain exposure limit (optimization (iii)). The power consumed $\left(p_{50}\right)$ by this network is approximately $5 \mathrm{~kW}$ higher and almost $20 \mathrm{~kW}$ lower than respectively the optimization towards power consumption and the optimization towards global exposure (Table 4). Analogously, the global exposure $\left(p_{50}\right)$ is about $10 \mathrm{mV} / \mathrm{m}$ lower and $164 \mathrm{mV} / \mathrm{m}$ higher.

When optimizing towards both power consumption and global exposure, a compromise is also obtained. The maximum power consumption is slightly higher than when optimizing only towards power consumption (100 kW versus $101 \mathrm{~kW}$ for $p_{50}$ in Table 4 , a differ- ence of only $1 \mathrm{~kW}$ ) but significantly lower than when optimizing towards global exposure $(121 \mathrm{~kW})$. Analogously, a global exposure of $163 \mathrm{mV} / \mathrm{m}\left(p_{50}\right)$ is found versus $268 \mathrm{mV} / \mathrm{m}$ and $104 \mathrm{mV} / \mathrm{m}$ when optimizing only towards power consumption and global exposure, respectively. The network obtained with the deployment tool here proposed performs even better than when optimizing towards power consumption while satisfying a certain exposure limit. For the considered case, the network consumes about $4 \mathrm{~kW}$ less (a reduction of 3.4\%) and has a $96 \mathrm{mV} / \mathrm{m}$ lower global exposure (a reduction of $37 \%$ ).

Table 4 lists the confidence interval for the power consumption $C I_{P C}$ and for the global exposure $C I_{E_{M}}$ calculated over the 40 simulations. These intervals are non-overlapping, so we can conclude that the obtained values for the different cases are significantly different.

Finally, note that this work studies the influence of the different optimizations on the network. When applying the algorithm in real life, it will use a sort of 'memory' about which base stations are already switched on which will constrain the number of switched base stations in a given period. In that case, the optimal solutions might not be obtained.

\section{Conclusion and future work}

In this paper, a capacity-based deployment tool is proposed for future green wireless access networks whereby three different levels of optimizations are possible: (i) towards power consumption, (ii) towards global exposure, (iii) towards power consumption while satisfying a certain exposure limit, and (iv) towards both power consumption and global exposure. By 'capacitybased', we mean that the network responds to the instantaneous bit rate required by the users active in the network. This can be done by using sleep mode for the 
base stations. The algorithms are applied on a realistic suburban area in Ghent, Belgium for LTE-Advanced. Comparing the different networks shows that optimizing the network towards power consumption results in a network with a low number of high-power base stations, while the network optimized towards global exposure consists of a high number of low-power base stations. These are clearly contradicting constraints when optimizing towards both power consumption and global exposure. A first compromise is obtained when optimizing the network towards power consumption while satisfying a certain exposure limit (here, $4.48 \mathrm{~V} / \mathrm{m}$ ). The power consumption $(101 \mathrm{~kW})$ and global exposure $(163 \mathrm{mV} / \mathrm{m})$ are in between the results of the optimizations towards power consumption $(100 \mathrm{~kW}$ and $268 \mathrm{mV} / \mathrm{m})$ and towards global exposure $(121 \mathrm{~kW}$ and $104 \mathrm{mV} / \mathrm{m}$ ). An even better compromise is obtained when using the algorithm here proposed for optimizing towards power consumption and global exposure. Compared to the first compromise, the power consumption is further reduced with $3.4 \%$, while the global exposure even decreases with $37 \%$. All users are served for all optimizations (user coverage $=100 \%$ ). Furthermore, the influence of the most important parameters of the algorithm is investigated. Choosing the appropriate weighting factors of the fitness function is important in order to obtain the desired results. The ratio of the two weighting factors is decisive in obtaining the right optimization. The higher (respectively the lower) this ratio, the more the network will be optimized towards global exposure (respectively power consumption). Increasing (respectively decreasing) the ratio $\frac{w_{1}}{w_{2}}$ with $50 \%$ results in a $1.2 \%$ higher (respectively lower) power consumption and a $6.7 \%$ lower (respectively higher) global exposure. Besides the weighting factors, it is also important to choose a realistic minimal distance between the base station and the human, especially one optimizing towards exposure. This parameter has limited influence on the results for power consumption. Finally, the distance between the grid points has no influence on the obtained results.

As future work, the uplink exposure will also be taken into account in our deployment tool. The goal is to optimize the network towards power consumption and both downlink and uplink exposure. In order to do this, an appropriate metric has to be considered.

\section{Acknowdlegment}

Emmeric Tanghe is a postdoctoral fellow of the Research Foundation Flanders (FWO-V).

\section{References}

[1] M. Deruyck, W. Joseph, L. Martens, Power consumption model for macrocell and microcell base stations, Transactions on Emerging Telecommunications Technologies, Vol. 25, No. 3, pp. 320-333, 2014.

[2] M.A. Marsan, L. Chiaraviglio, D. Ciullo, and M. Meo, A Simple Analytical Model for the Energy-Efficient Activation of Access Points in Dense WLANs, 1st International Conference on Energy-Efficient Computing and Networking (e-Energy), Passau, Germany, pp. 159-168, 2010.

[3] G. Koutitas, Green Network Planning of Single Frequency Networks, IEEE Transactions on Broadcasting, Vol. 56, No. 4, 2010, pp. 541-550.

[4] TNS Opinion \& Social, Eurobarometer 73.3, Electromagnetic fields, June 2010

[5] M. Tesanovic, E. Conil, A. De Domenico, R. Agüero, F. Freudenstein, L.M. Correia, S. Bories, L. Martens, P.M. Wiedemann, and J. Wiart, The LEXNET project, IEEE Vehicular Technology Magazine, Vol. 9, No. 2, pp. 20-28, 2014.

[6] B. Vanhaelewyn, G. Pauwels, M. Maes, L. De Marez, Measuring Digital Media Trends in Flanders, Digimeter augustusseptember 2014, online: http://www.iminds.be/nl/inzicht-indigitale-technologie/digimeter.

[7] D. Plets, W. Joseph, K. Vanhecke, L. Martens, Exposure Optimization in Indoor Wireless Networks by Heuristic Network Planning, Progress in Electromagnetics Research - PIER, Vol. 139, 2013, pp. 445-478.

[8] O. Arnold, F. Richter, G. Fettweis, O. Blume, Power Consumption Modeling of Different Base Station Types in Heterogeneous Cellular Networks, Future Network and Mobile Summit (FuNeMS), pp. 1-8, 2010.

[9] C. Desset, B. Debaillie, V. Giannini, A. Fehske, G. Auer, H. Holtkamp, W. Wajda, D. Sabella, F. Richter, M. J. Gonzalez, H. Klessig, I. Gódor, M. Olsson, M.A. Imran, A. Ambrosy, O. Blume, Flexible power modeling of LTE base stations, IEEE Wireless Communications and Networking Conference (WCNC), pp. 2858-2862, 2012.

[10] Y. Zhang, L. Budzisz, M. Meo, A. Conte, I. Haratcherev, G. Koutitas, L. Tassiulas, M.A. Marsan, S. Lambert, An Overview of Energy-efficient Base Station Management Techniques, 24th Tyrrhenian International Workshop on Digital Communications - Green ICT (TIWDC), pp. 1-6, 2013.

[11] F.G. Debele, N. Li, M. Meo, M. Ricca, Y. Zhang, Experimenting Resource-on-Demand Strategies for Green WLANs, ACM SIGMETRICS Performance Evaluation Review, Vol. 42, No. 3, 2014, pp. 61-66.

[12] F. Ganji, L. Budzisz, F. G. Debele, N. Li, M. Meo, M. Ricca, Y. Zhang, A. Wolisz, Greening campus WLANs: Energyrelevant usage and mobility patterns, Elsevier Computer Networks, December 2014, DOI: 10.1016/j.comnet.2014.10.033.

[13] I. Ashraf, F. Boccardi, L. Hester, Power Savings in Small Cell Deployments via Sleep Mode Techniques, 21st IEEE International Symposium on Personal, Indoor and Mobile Radio Communications Workshops (PIMRC Workshops), pp. 307311, 2010.

[14] R. Litjens, L. Jorguseshi, Potential of Energy-Oriented Network Optimisation: Switching Off Over-Capacity in Off-Peak Hours, 21st IEEE International Symposium on Personal, Indoor and Mobile Radio Communications (PIMRC), pp. 1658-1662, 2010.

[15] M.W. Arshad, A. Vastberg, T. Edler, Energy Efficiency Gains Through Traffic Offloading and Traffic Expansion in Joint Macro Pico Deployment, IEEE Wireless Communications and Networking Conference (WCNC), pp. 2230-2235, 2012.

[16] A.B. Sakh, Ö. Bulakci, S. Redana, B. Raaf, J. Hämäläinen, Eval- 
uating the energy efficiency of LTE-Advanced relay and picocell deployments, IEEE Wireless Communications and Networking Conference (WCNC), pp. 2362-2367, 2012.

[17] P. Chandhar, S.S. Das, Area Energy Efficiency Analysis for OFDMA femtocell networks, 11th International Symposium on Wireless Communications Systems (ISWCS), pp. 771-775, 2014.

[18] www.lexnet-project.eu

[19] M. Deruyck, W. Joseph, E. Tanghe, L. Martens, Reducing the Power Consumption in LTE-Advanced Wireless Access Networks by a Capacity Based Deployment Tool, Radio Science, Vol. 49, No. 9, pp. 777-787, 2014.

[20] COST telecommunications, COST Action 231: Digital mobile radio towards future generation systems, European Commission, 1999.

[21] M. Deruyck, E. Tanghe, W. Joseph, W. Vereecken, M. Pickavet, L. Martens, B. Dhoedt, Model for power consumption of wireless access networks, IET Science, Measurement and Technology, Vol. 5, No. 4, pp. 155-161, 2011.

[22] M.J. Gonzalez, D. Ferling, W. Wajda, A. Erdem, P. Maugars, Concepts for Energy Efficient LTE Transceiver Systems in Macro Base Stations, Future Network \& Mobile Summit, Warsaw, Poland, pp. 1-8, 2011.

[23] International Commission on Non-ionizing Radiation Protection (ICNIRP), Guidelines for limiting exposure to time-varying electric, magnetic, and electromagnetic fields (up to $300 \mathrm{GHz}$ ), Health Physics, Vol. 74, No. 4, pp. 494-522, April 1998.

[24] D. Urbinello, W. Joseph, A. Huss, L. Verloock, J. Beekhuizen, R. Vermeulen, L. Martens, M. Röösli, Radio-frequency electromagnetic field (RF-EMF) exposure levels in different European outdoor urban environments in comparison with regulatory limits, Elsevier Environment International, Vol. 68, 2014, pp. 4954. 